\title{
XII. Modos ventilatorios en ventilación no invasiva
}

\author{
GERARDO FERRERO R.*
}

\section{Ventilatory modes for noninvasive ventilation}

Se hará una revisión de los diferentes modos o modalidades ventilatorios aplicados en la actualidad en ventilación no invasiva (VNI), tanto para los pacientes con insuficiencia respiratoria aguda como crónica. Se describirán, a su vez, actualizaciones desde el punto de vista tecnológico en relación a los sistemas de compensación de fuga, disparo y ciclado automático.

\section{Presión positiva continua en la vía aérea}

La presión positiva continua en la vía aérea (CPAP) es un modo de operación del ventilador, de un generador de alto flujo o de un compresor portátil, donde el paciente respira espontáneamente dentro de un nivel de presión superior a la atmosférica (Figura 1). Cualquiera sea el sistema empleado, éste debe ser capaz de generar altos flujos (entre 40-120 L/min) para satisfacer la demanda inspiratoria del paciente con insuficiencia respiratoria aguda.

La CPAP es, por lo tanto, una modalidad de ventilación espontánea, controlada por presión, gatillada por flujo o presión, limitada por presión, y ciclada por el paciente. Como la ventilación no es apoyada, el volumen corriente (VT), la frecuencia respiratoria y consecuentemente, la ventilación alveolar, pueden variar en el tiempo ya que dependen del patrón respiratorio del paciente.

La CPAP permite generar un gradiente de presión positivo dentro del ciclo inspiración-espiración. El efecto de la presión positiva sobre ambas fases del ciclo respiratorio permite aumentar la presión media de la vía aérea, con el consecuente aumento de la capacidad residual funcional (CRF), y disminución del trabajo respiratorio en situaciones clínicas donde exista una reducción de la CRF. A su vez, desde un punto de vista hemodinámico, dicha presión positiva constante intratorácica determina una caída del retorno venoso, lo permite que la CPAP sea un modo de apoyo en la descompensación respiratoria por edema pulmonar agudo. En esta condición, la CPAP produce un rápido alivio de la disnea y mejoría del intercambio gaseoso comparado con la respiración espontánea ${ }^{1}$, siempre que no exista hipercapnia ya que, como se ha dicho, esta modalidad no aumenta la ventilación ${ }^{2,3}$.

\section{Ventilación asistido-controlada por volumen}

La ventilación asistido-controlada por volumen (A/CMV) es una modalidad en la que los ciclos mandatorios son proporcionados al paciente a una determinada frecuencia, volumen y flujo. Entre los ciclos mandatorios, el paciente puede gatillar el ventilador, recibiendo un ciclo asistido a un volumen prefijado. Los ciclos mandatorios son gatillados por tiempo, mientras que los ciclos asistidos son gatillados por flujo o presión, respondiendo al esfuerzo inspiratorio del paciente. Ambas modalidades son limitadas por flujo y cicladas por volumen (Ver Equipos e Interfaces para una definición de estos términos).

La onda de flujo es de tipo continuo o rectangular, aunque algunos ventiladores permiten la opción de proporcionar una onda de tipo desacelerado regulada por software. Esta última es la recomendada para la aplicación de VNI, ya que es más confortable para el paciente.

Esta modalidad es de primera elección en el caso de la VNI crónica domiciliaria en aquellos pacientes con enfermedades neuromusculares avanzadas. En tales pacientes, esta modalidad permite asistir la tos, ya que aumenta la capaci-

Kinesiólogo-Fisiatra, Hospital de Rehabilitación Respiratoria María Ferrer, Buenos Aires, Argentina. Fellow Internacional American Association for Respiratory Care (AARC). 
dad inspiratoria y consecuentemente el flujo máximo de tos. También es una alternativa razonable cuando no se dispone de un ventilador que ofrezca modalidades ventilatorias más sofisticadas y confortables para el paciente (Figura 2).

Los siguientes ajustes de las variables de programación en el modo A/CMV se recomiendan:

\section{a) Ajuste de volumen corriente}

Si la enfermedad de base cursa con un aumento de la resistencia de la vía aérea y de la
CRF, se recomienda emplear un VT entre 5-7 $\mathrm{mL} / \mathrm{kg}$, suficiente para satisfacer la demanda ventilatoria sin aumentar o promover una mayor presión positiva intrínseca de final de espiración (PEEPi). La aplicación concomitante de presión positiva de final de espiración (PEEP) es usual en estos casos, con el fin de contrabalancear la PEEPi del paciente con enfermedad pulmonar obstructiva crónica (EPOC), si bien no es la modalidad de elección para aplicar $\mathrm{VNI}^{4}$. Sin embargo, es bien sabido que la ventilación me-

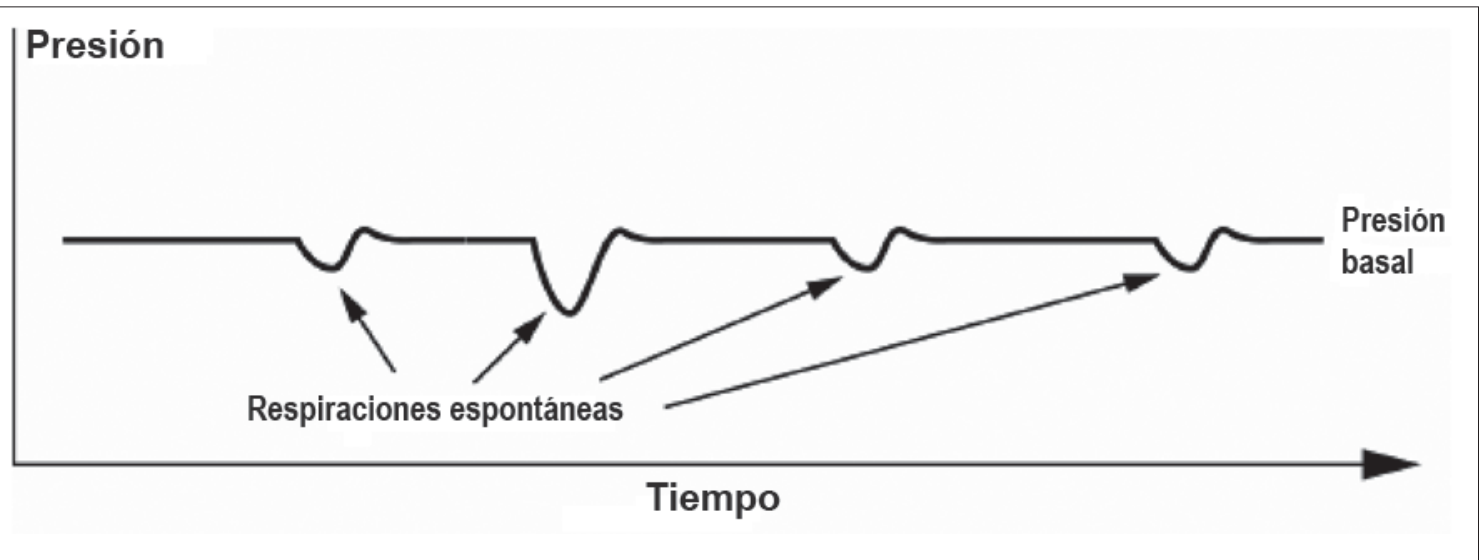

Figura 1. Presión positiva continua en la vía aérea (CPAP). La caída de la presión durante la fase inspiratoria es dependiente del esfuerzo inspiratorio del paciente dentro de un sistema de presión elevada sobre la atmosférica.

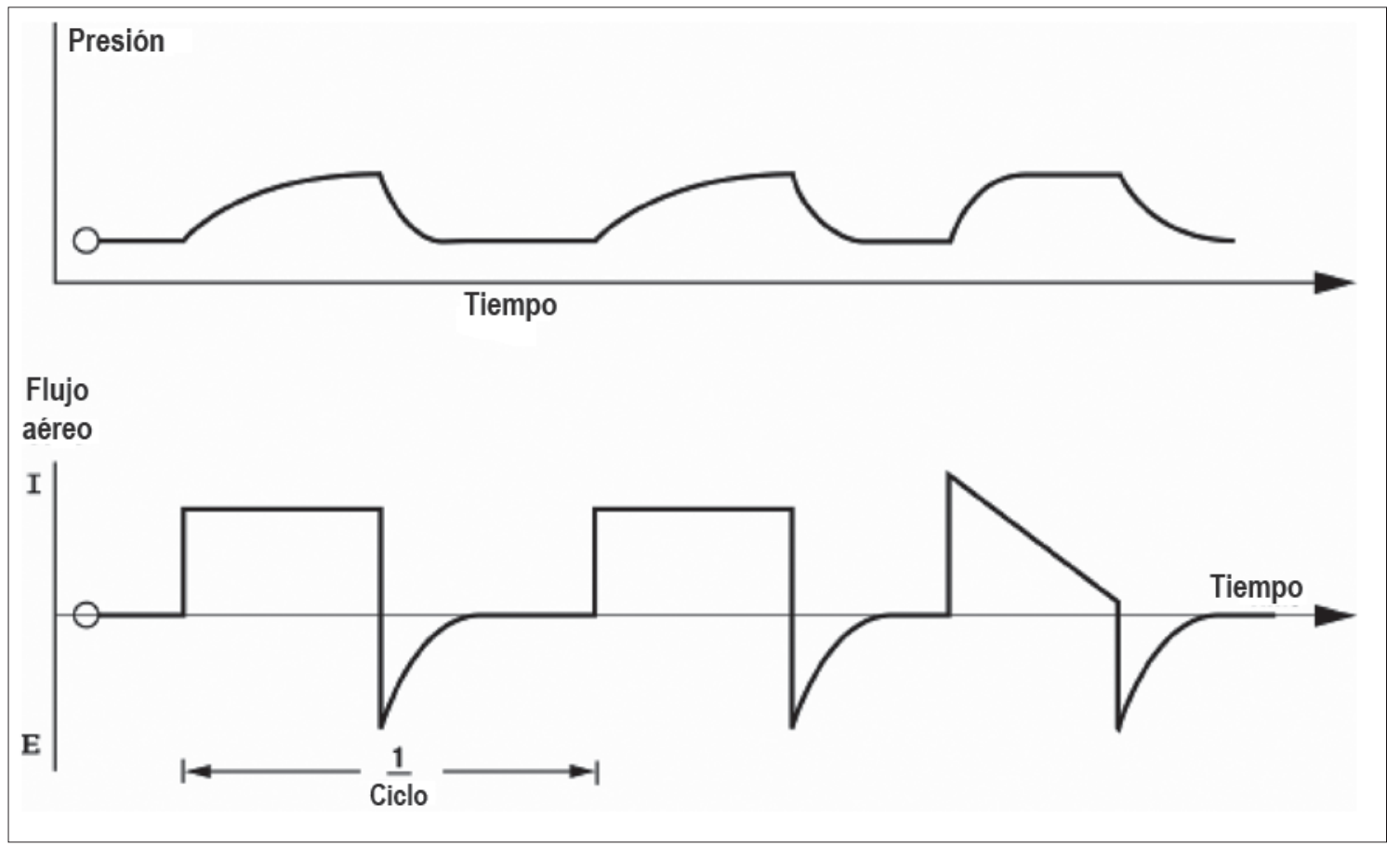

Figura 2. Modo de ventilación mecánica controlada por volumen (CMV). En los primeros dos ciclos la onda de flujo es cuadrada, mientras en el último ciclo es desacelerada. Esta última permite sostener la presión en forma sostenida comparada con la onda cuadrada. 
cánica empleando VT bajos $(5-7 \mathrm{~mL} / \mathrm{kg})$, sea en forma espontánea o mecánica, promueve el desarrollo de microatelectasias, con el consiguiente descenso de la distensibilidad pulmonar y aumento del cortocircuito intrapulmonar en aquellas enfermedades que provocan un marcado aumento de la elastancia (por ejemplo, cifoescoliosis $)^{5,6}$. Dado que los pacientes con enfermedades neuromusculares presentan distensibilidad tóraco-pulmonar reducida, se sugiere en tales casos el uso de VT entre $8-12 \mathrm{~mL} / \mathrm{kg}$, previniendo así las microatelectasias ${ }^{7}$, facilitando la capacidad de fonación, tos y expectoración en este grupo de pacientes. El empleo de VT relativamente elevados permite, a su vez, compensar el volumen de fuga que puede ocurrir alrededor de la máscara o pieza bucal ${ }^{8}$.

\section{b) Ajuste del flujo inspiratorio máximo}

El flujo inspiratorio proporcionado por el ventilador mecánico es un determinante importante del trabajo respiratorio de un paciente dado. Si se selecciona un flujo inspiratorio que esté por debajo del flujo inspiratorio demandado por el paciente, éste incrementará su esfuerzo inspiratorio, aumentando así la desadaptación del ventilador y el trabajo respiratorio9. Consecuentemente, el flujo a seleccionar debe satisfacer la demanda del paciente. Se pueden requerir valores entre $60-80 \mathrm{~L} / \mathrm{min}$ en las descompensaciones de la EPOC y de 40-60 L/min en aquellos cuadros en que la demanda es menos marcada, como ocurre en los pacientes con enfermedades neuromusculares.

\section{Ventilación asistido-controlada con control de presión}

La ventilación con control de presión (PCV; pressure control ventilation) se refiere a aquella en la cual los disparos son gatillados por tiempo o por el paciente (en el modo asistido, también llamado Presión de Asistencia) limitados por presión (presión inspiratoria) y ciclados por tiempo (tiempo inspiratorio). La onda de flujo es de tipo desacelerado; el VT va a ser dependiente de la impedancia tóraco-pulmonar que presente el paciente: a mayor impedancia, la presión inspiratoria máxima se obtendrá más rápidamente y consecuentemente el VT será menor.

Con la ventilación por presión, la presión inspiratoria máxima es la variable a programar, a diferencia del modo volumétrico en que se programa el VT. Adicionalmente, el operador debe fijar una frecuencia respiratoria mínima, un tiempo inspiratorio (Ti) o relación Inspiración: Espiración y el nivel de sensibilidad del gatillado $^{10}$.

Las diferencias entre el modo controlado por presión y el modo controlado por volumen son la consistencia de la presión inspiratoria máxima en uno y del VT en el otro. Así, con la ventilación con control de presión la presión máxima es constante pero el VT puede variar. La principal ventaja de esta modalidad para aplicarla durante la VNI es que el flujo es variable, capaz de ajustarse a la demanda del paciente, dentro de un delta de presión preestablecido. A mayor delta de presión, mayor capacidad para generar flujos. Este modo es de elección cuando el paciente no logra una adecuada adaptación al ventilador en modalidad de presión de soporte (PSV), por fugas alrededor de la máscara. En PCV, al ser el tiempo inspiratorio fijo, independientemente de la fuga del sistema, el ciclado a espiración puede ajustarse mejor al ciclado deseado por el paciente (Figura 3) ${ }^{11}$. El operador fija el tiempo inspiratorio del ventilador en función del tiempo inspiratorio del paciente, empleando habitualmente tiempos entre 0,8-1,2 segundos, dependiendo de la enfermedad de base.

\section{Ventilación con presión de soporte (PSV)}

La modalidad de Presión de Soporte (PSV) es una forma de ventilación asistida, en la que el paciente dispara el ventilador respiración a respiración. El operador decide que nivel de presión se debe suministrar, la cual será mantenida durante todo el ciclo inspiratorio espontáneo del paciente. El mismo se mantiene gracias a un sistema de flujo desacelerado y servo controlado, a diferencia de la ventilación a presión positiva inspiratoria (IPPB), que es ciclada por presión y por lo tanto la inspiración termina cuando se ha alcanzado una presión prefijada. La PSV es ciclada por flujo, permitiendo al paciente regular su tiempo inspiratorio, tiempo espiratorio, flujo inspiratorio, VT y volumen minuto. Como resultado se obtiene un mayor confort y menor trabajo respiratorio ${ }^{12}$. La PSV combinada con PEEP, es la modalidad más corrientemente usada en la aplicación de VNI, principalmente durante la insuficiencia respiratoria hipercápnica de pacientes con EPOC (Figura 4) ${ }^{13}$.

Al ser la PSV un modo asistido, necesita obligatoriamente de la actividad espontánea del paciente, de manera que pueda gatillar el ventilador. El gatillado puede ser por flujo, presión o automáticamente de acuerdo a la forma de la 
onda de flujo (Auto Trak®). La PSV puede ser así definida como un sistema asistido, limitado por presión y ciclado por flujo. El cese de la inspiración puede producirse en función de un porcentaje del flujo máximo alcanzado (por ejemplo, a $25 \%$ del pico de flujo inspiratorio), o bien a valores predeterminados del mismo (por ejemplo, cuando el flujo inspiratorio ha disminuido a
$5 \mathrm{~L} / \mathrm{min})$, dependiendo del ventilador. El límite de tiempo para la duración de la inspiración es importante en caso de una despresurización del sistema, como ocurre cuando se produce una gran fuga en el circuito. En estas circunstancias el ventilador no puede alcanzar la presión de trabajo programada, y al mantener un flujo inspiratorio elevado tratando de lograrlo, no se

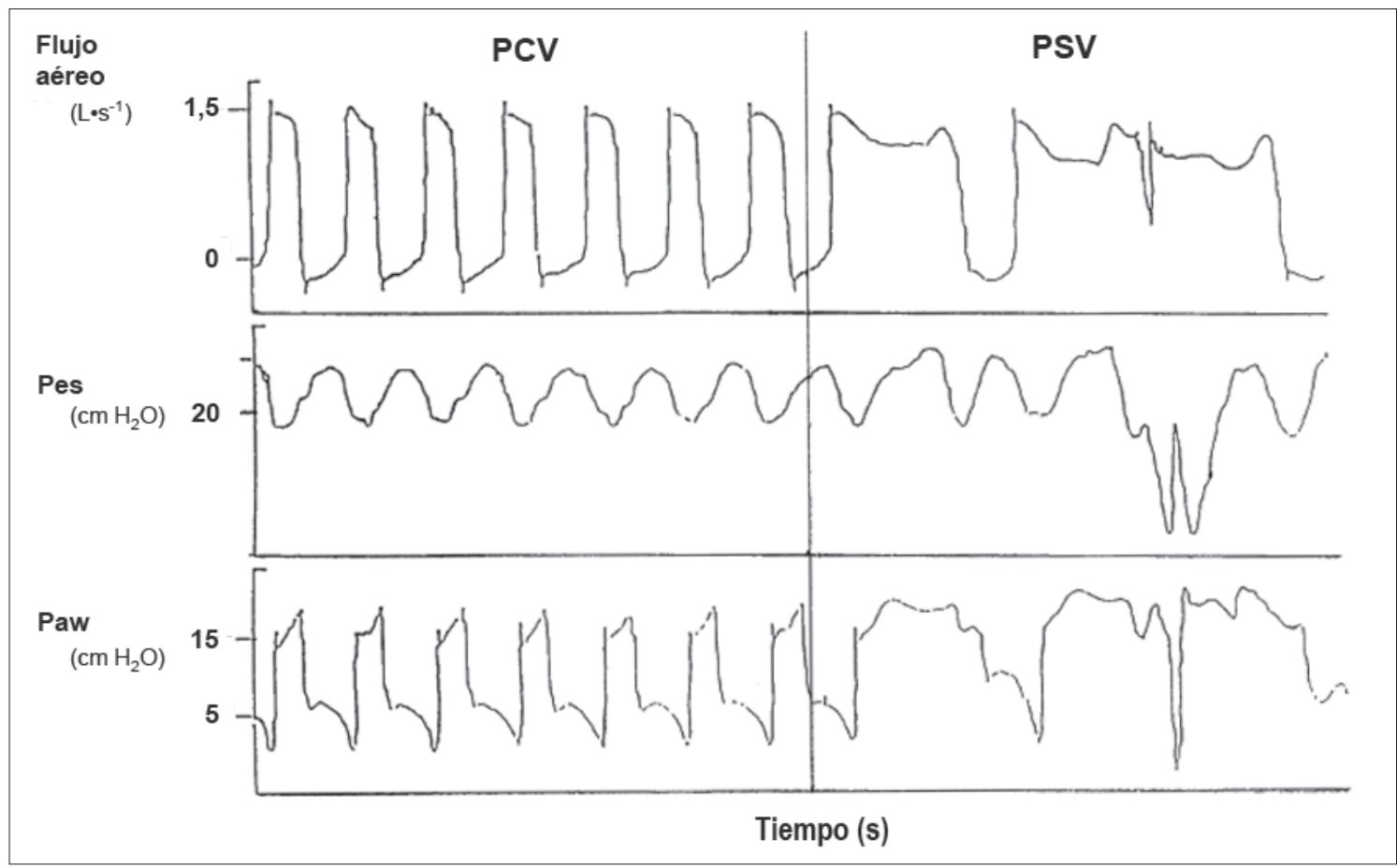

Figura 3. Flujo, presión esofágica (Pes) y presión de la vía aérea (Paw) durante la aplicación de ventilación no invasiva en la modalidad controlada por presión (PCV; izquierda) y en la modalidad de presión de soporte (PSV; derecha). El patrón irregular y asincrónico en PSV se debe a un problema del ciclado por flujo de esta modalidad. La asincronía se corrige con el modo PCV, que es ciclado por tiempo. Modificado de Calderini y cols ${ }^{11}$.

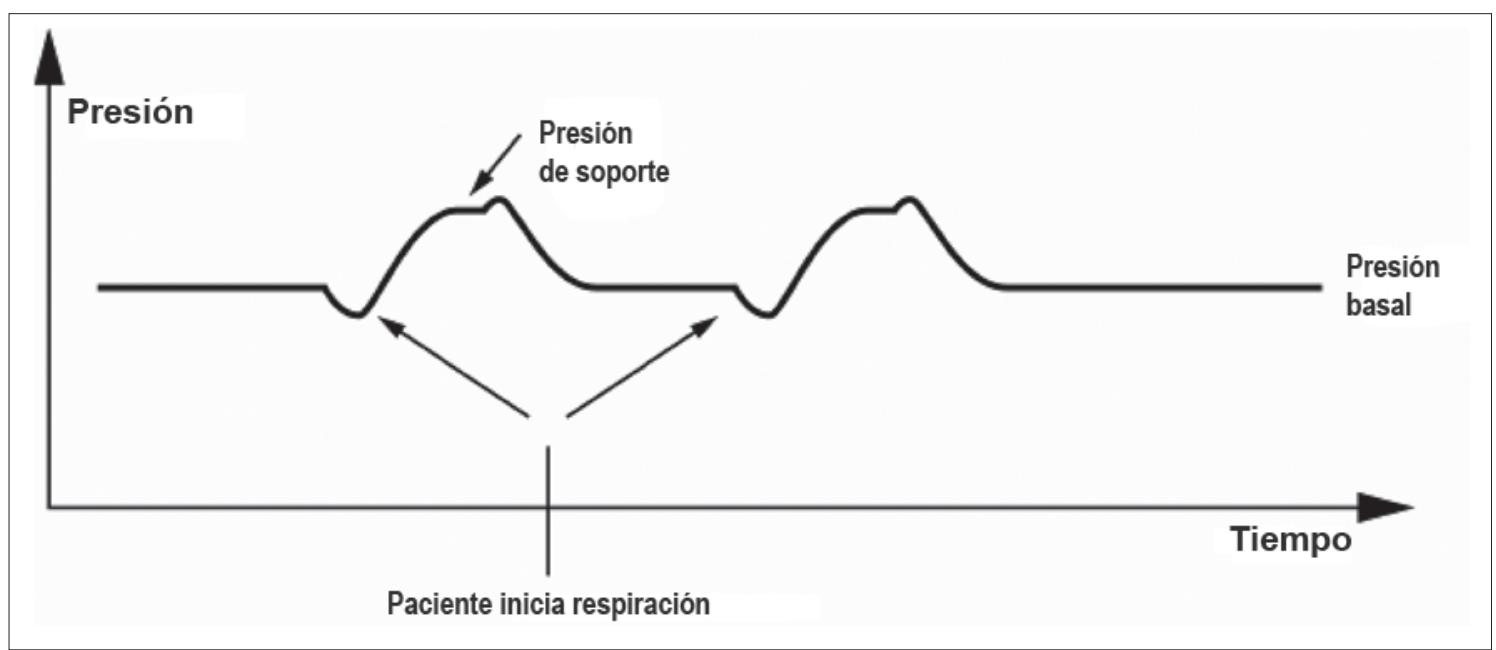

Figura 4. Modalidad de presión de soporte asociada a presión positiva de final de espiración (PEEP). 
cumple el criterio de flujo para iniciar la espiración, prolongándose excesivamente la inspiración. Por ello, los ventiladores cuentan con un sistema de corte de flujo inspiratorio que se produce cuando la inspiración se ha prolongado aproximadamente 3 a 4 segundos, como un mecanismo de seguridad para evitar la sobre insuflación ${ }^{14}$. Sin embargo, dicho tiempo inspiratorio en exceso puede promover la desadaptación y fracaso de la VNI (ver Figura 3$)^{11}$.

La fase espiratoria es libre de asistencia, pudiendo agregarse una cierta PEEP para contrabalancear la PEEPi en el caso de pacientes con EPOC. En los pacientes con enfermedades restrictivas, la misma PEEP permite aumentar la CRF por reclutamiento alveolar, disminuyendo así el trabajo respiratorio.

Para determinar el nivel adecuado de presión inspiratoria durante la PSV se han descrito diferentes métodos. MacIntyre utilizó el máximo nivel de PSV que resultara en una condición de patrón respiratorio estable, sin fases de hiper o hipopnea ${ }^{12}$. Brochard ha recomendado observar el nivel de reclutamiento de los músculos accesorios de la inspiración principalmente el esternocleidomastoideo a través de la inspección y palpación ${ }^{15}$. Diversos autores concuerdan, por otro lado, que la frecuencia respiratoria es un modo sencillo y práctico para verificar el nivel adecuado de PSV, siendo valores entre 15-25 respiraciones/min los recomendados.

La PSV permite seleccionar la velocidad de presurización de la vía aérea, denominada usualmente rampa de presurización, rise time o pressure slope. Corresponde al tiempo que transcurre entre el disparo del paciente y el momento

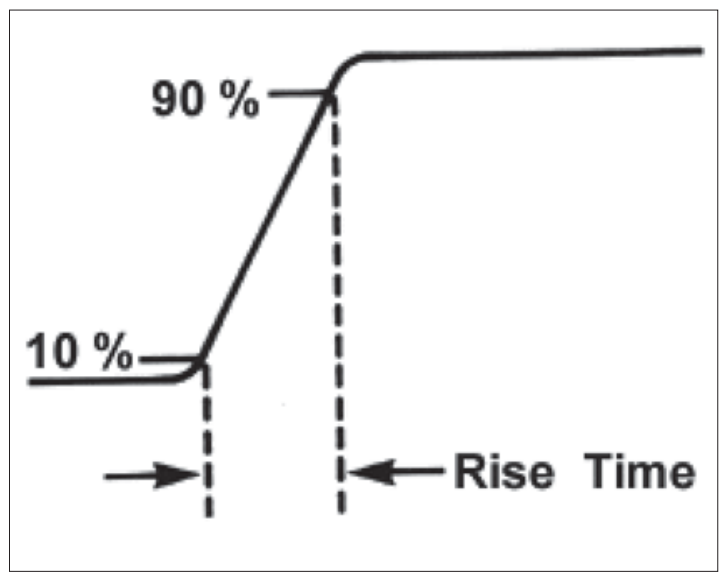

Figura 5. Rampa de presurización o Rise Time. Corresponde al tiempo que transcurre desde el inicio de la inspiración por parte del paciente hasta alcanzar la presión de soporte establecida. en que se alcanza la presión inspiratoria establecida. Cuanto mayor sea la demanda de flujo por parte del paciente, más rápida será la necesidad de presurizar su vía aérea. En tales casos se programará una rampa de 0,05 a 0,1 segundos. A medida que el paciente se estabiliza, se puede cambiar a tiempos más prolongados $(0,3-0,4$ segundos). La rampa de presurización es, entonces, un criterio de confort inspiratorio para el paciente, tanto agudo como crónico durante la aplicación de VNI (Figura 5).

En algunos pacientes pueden presentarse signos de asincronía paciente-ventilador entre la respiración espontánea y la entrega de flujo por parte del ventilador. Es en estos casos donde se debe monitorear si la presión de soporte prefijada se ajusta a las variables de ventilación que solicita el paciente.

La sincronización ventilador-paciente puede ser optimizada en el modo PSV de las siguientes formas ${ }^{21}$ :

- Aumentando la sensibilidad del gatillado, sin provocar autociclado. En aquellos ventiladores que lo dispongan, esto puede lograrse utilizando la sensibilidad inspiratoria-espiratoria automática (Auto Trak®).

- Asegurar las características de la interface, evitando fugas alrededor de la máscara en los ventiladores sin compensación de fuga, o mayor a $20-30 \mathrm{~L} / \mathrm{min}$ en los sistemas de flujo continuo con compensación de fugas.

- Minimizando el tiempo de alcance de presión máxima, es decir, reduciendo el tiempo de rampa.

- Reduciendo el nivel de presión de soporte al mínimo indispensable para evitar aumento de la PEEPi en pacientes con EPOC.

- Aumentando el nivel de sensibilidad de terminación de la fase inspiratoria en aquellos ventiladores que lo dispongan.

- Evitar la colocación de elementos interpuestos que incrementen la resistencia de la vía aérea (por ejemplo, nariz artificial, filtros antibacterianos, etc). En caso de estar incluidos en el circuito del ventilador, aumentar la PSV para compensar la misma.

- Asistencia en la tos y remoción de secreciones bronquiales a través de fisioterapia respiratoria.

- Tratamiento broncodilatador.

\section{Modo binivel (IPAP/EPAP)}

El modo binivel es un análogo cualitativo, pero no cuantitativo, de la PSV + PEEP. La presión de soporte es la resultante de la diferencia entre la IPAP y la EPAP. Tómese en cuenta que EPAP $=$ PEEP, por lo que toda modificación de la 
EPAP sin cambio proporcional de la IPAP, implica aumento o disminución de la PSV (Figura 6) ${ }^{22}$. Para más detalles se sugiere leer el capítulo sobre Equipos e Interfaces.

La nomenclatura para esta modalidad es la siguiente:

Modo $S$ (Spontaneous): El ventilador suministra soporte de presión binivel. La unidad activa la presión positiva (IPAP) en respuesta a un esfuerzo inspiratorio espontáneo y cicla a presión positiva espiratoria (EPAP) durante la espiración.

Modo S/T (Spontaneus-Timed): Es seme- jante el modo $\mathrm{S}$, salvo que puede además administrar una respiración iniciada mecánicamente si el paciente no respira espontáneamente en un tiempo prefijado (frecuencia de reaseguro o de respaldo). El modo $\mathrm{S} / \mathrm{T}$ garantiza a los pacientes un número mínimo de respiraciones basado en el valor de programación de la frecuencia respiratoria. Si el paciente no inicia una respiración en el tiempo determinado por el control de la frecuencia, la unidad inicia una respiración disparada y ciclada por tiempo (tiempo inspiratorio), limitada por presión al nivel de IPAP preestablecido (Figura 7).

\section{PS + PEEP vs. BILEVEL}

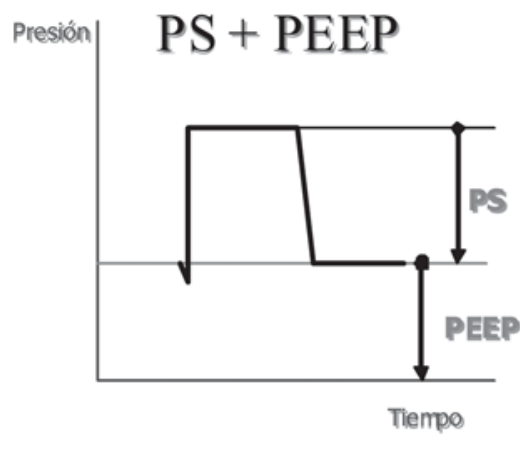

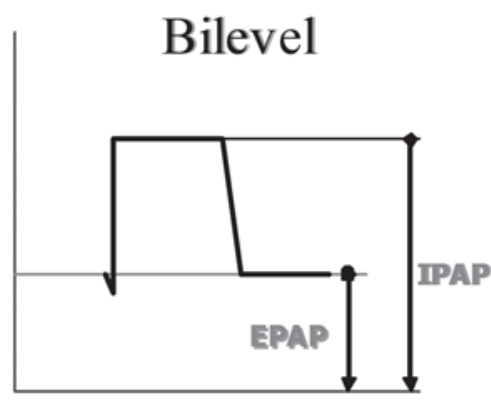

$P S=I P A P-E P A P$
Figura 6. Modalidad binivel de un ventilador de flujo continuo (derecha) comparada con la modalidad de presión de soporte (PSV) asociada a presión positiva de final de espiración (PEEP). Para más detalles ver texto. IPAP: presión inspiratoria de vía aérea positiva; EPAP: presión espiratoria positiva.

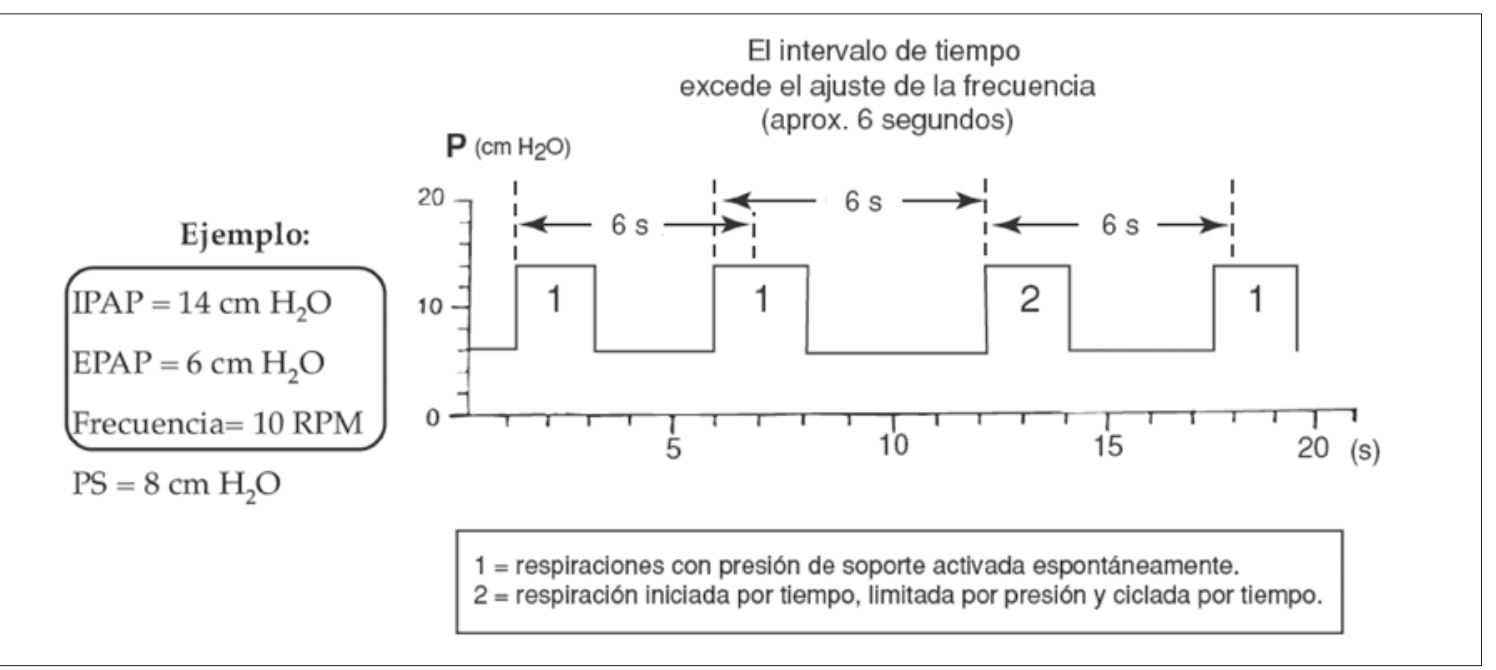

Figura 7. Modos Espontáneo y Espontáneo-Ciclado por Tiempo de la ventilación con presión binivel. 


\section{Presión de soporte con volumen promedio asegurado}

La presión de soporte con volumen promedio asegurado (AVAPS; average volume-assured pressure support ventilation) es una modalidad dual o híbrida que permite mantener un VT consistente, como en la ventilación controlada por volumen, con las ventajas de la presión de soporte. Esto se logra mediante un control automático de la IPAP entregada al paciente. El software del ventilador ajusta la IPAP entre un rango de presiones mínima (IPAP mínimo) y máximo (IPAP máximo), con cambios muy graduales respiración a respiración, para evitar percepción brusca de dicho cambio y disconfort. Si el esfuerzo inspiratorio del paciente disminuye, la modalidad AVAPS aumenta de manera automática la IPAP para mantener el VT prefijado (Figura 8). El nivel de IPAP no sobrepasará la IPAP máxima programada, aunque el VT prefijado no fuese alcanzado. En este último caso, la alarma de bajo VT se activa, debiéndose modificar el límite superior de IPAP para alcanzar el VT programado, o cambiar a otra modalidad como PCV, utilizando menor presión pero mayor tiempo inspiratorio ${ }^{26}$.

\section{Ventilación proporcional asistida}

La ventilación proporcional asistida (PAV) fue desarrollada como un modo de mejorar la res- puesta del ventilador a la demanda inspiratoria y espiratoria del paciente, basado en una adaptación respiración a respiración. Al igual que la PSV, la PAV es una modalidad espontánea, iniciando el disparo el propio paciente. Sin embargo, a diferencia de PSV, que está limitada por una presión inspiratoria ajustada por el operador, la PAV provee flujo y presión proporcional al esfuerzo inspiratorio espontáneo del paciente. Esta característica determina que tanto el inicio, la amplitud y la finalización del ciclo inspiratorio sea regulado totalmente por el paciente (Figura 9).

La amplificación de la señal inspiratoria del paciente está basada en la lectura del flujo del mismo, con un sistema retroalimentado que permite respetar así el patrón respiratorio del paciente. Se entiende, por lo tanto, que el ciclado a espiración no depende de una caída de flujo específica como ocurre en la PSV, sino que se produce cuando el paciente inicia espontáneamente la fase espiratoria. Las variables citadas permiten lograr una máxima sincronía entre el paciente y ventilador, como ha sido bien demostrado $^{27-29}$.

\section{Variables comunes a los diferentes modos}

\section{Sensibilidad del gatillo}

El gatillado o trigger es de dos tipos básicos: por presión o por flujo. En el sistema de disparo por presión, el paciente debe vencer una resis-

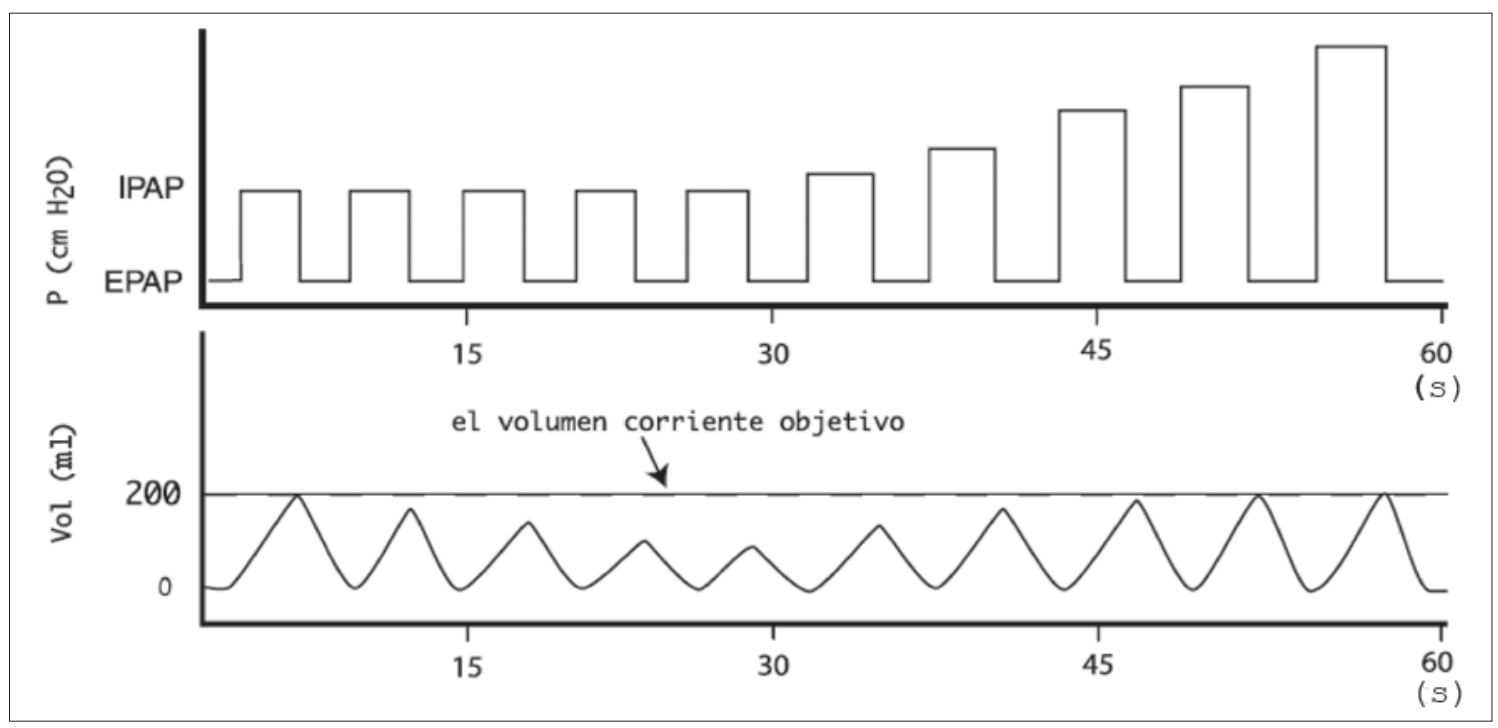

Figura 8. Modalidad de presión de soporte con volumen promedio asegurado (AVAPS). Nótese que la presión inspiratoria positiva (IPAP) debe aumentar para mantener un volumen corriente constante prefijado (200 mL en este ejemplo). 


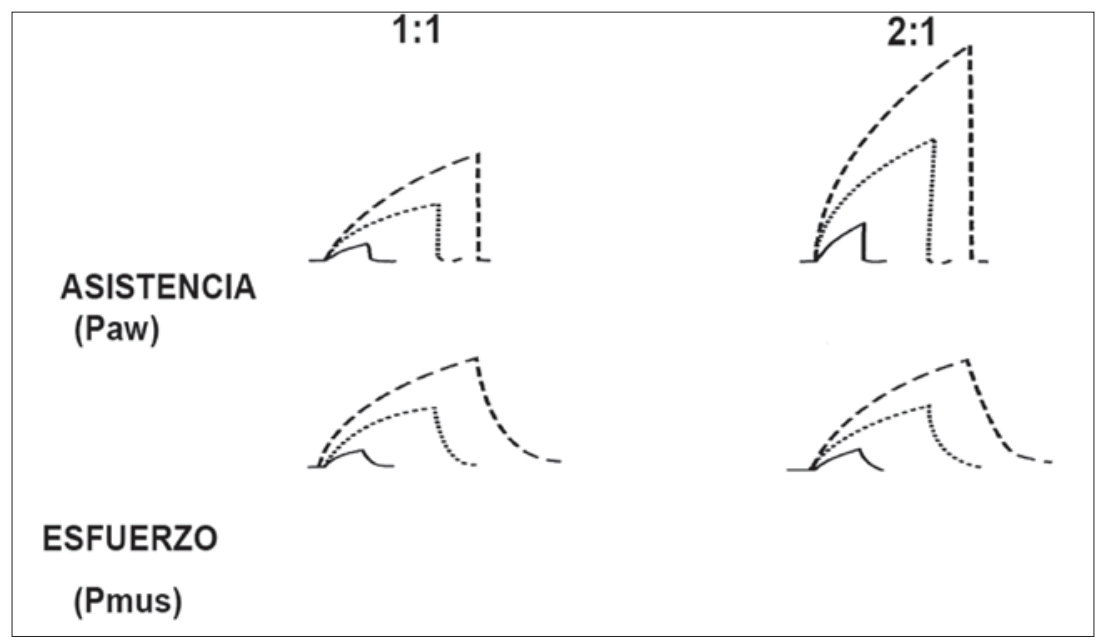

Figura 9. Ventilación Proporcional Asistida. Nótese la amplificación de la señal de presión en función del esfuerzo del paciente. Para más detalles ver el texto. Paw: presión positiva en la vía aérea; Pmus: esfuerzo músculos respiratorios. tencia umbral (sensibilidad) para que el ventilador entregue el flujo de aire programado. Dicha presión negativa debe ser la mínima $(-0,5$ a $-1,0$ $\mathrm{cm} \mathrm{H}_{2} \mathrm{O}$ ), aumentando así la sensibilidad del disparo, el cual provocará disminución del trabajo respiratorio. Si el mismo se colocara en presiones más elevadas $\left(-2 /-5 \mathrm{~cm} \mathrm{H}_{2} \mathrm{O}\right)$, el paciente debería hacer más esfuerzo inspiratorio, con el consiguiente incremento del trabajo respiratorio y mayor desadaptación al ventilador ${ }^{31}$.

En función a lo citado en el primer párrafo, entre el disparo del paciente y la entrega del flujo por parte del ventilador, encontramos una pausa la cual tendrá relación con la sensibilidad programada y el tipo de ventilador utilizado: a menor sensibilidad, mayor tiempo de pausa ${ }^{32}$.

El sistema de demanda por flujo consta de un circuito con flujo colateral (flow-by) programado por el operador, habitualmente en un rango entre 3-20 L/min, con un ajuste de sensibilidad de gatillo entre 1-5 L/min. Cuando el neumotacógrafo situado en la válvula espiratoria detecta un cambio en el flujo colateral igual al ajuste de la sensibilidad, se activa la inspiración. En la actualidad, tanto el disparo por presión o por flujo no muestran diferencias en lo referente al retardo inspiratorio, en virtud del avance tecnológico en los ventiladores, sin embargo, debe analizarse cada caso en particular, tanto el tipo de paciente como el ventilador aplicado.

Los ventiladores portátiles o de flujo continuo trabajan con disparo por flujo. El sistema más sofisticado es el Auto Trak® (Respironics Inc., Murrysville, USA), donde un sistema de análisis multiparamétrico permite compensar la fuga, y generar el disparo inspiratorio y el ciclado espiratorio en forma totalmente automática. Dicho algoritmo, a su vez, permite un menor retardo inspiratorio y un producto presión-tiempo disminuido en relación a otros ventiladores, aún comparado con aquellos de cuidados intensivos (Figura 10) $)^{33-35}$.

\section{Presión positiva de final de espiración (PEEP)}

La PEEP aplicada en la ventilación no invasiva cumple los mismos criterios fisiológicos que cuando es aplicada en ventilación invasiva convencional. La ventaja mecánica de la PEEP, se diferencia como la capacidad de aumentar la $\mathrm{CRF}$, con la consiguiente mejoría de la oxigenación $^{36}$. Por otro lado, la implicancia sobre la PEEPi y su contrabalanceo (Figura 11), es vital durante la VNI en pacientes con EPOC descompensados. La combinación entre PSV + PEEP permite disminuir el trabajo respiratorio y el consumo de $\mathrm{O}_{2}$ diafragmático en este grupo de pacientes, comparado con la CPAP o PSV utilizados en forma independiente ${ }^{13}$.

Como se comentó previamente en este capítulo, la presión positiva intratorácica dada por la PEEP/CPAP disminuye el retorno venoso, reduciendo funcionalmente la poscarga del ventrículo izquierdo. Esta variable fisiológica es la base de la aplicación de CPAP o PSV + PEEP en el edema pulmonar agudo, permitiendo así una redistribución del líquido intrapulmonar ${ }^{36}$. La PEEP es de vital utilidad cuando es aplicada como coadyuvante de cualquier modo ventilatorio, salvo CPAP o APRV, donde la PEEP está implícita durante la VNI.

\section{Conclusiones}

Los modos controlados o con ventilación de reaseguro deben ser elegidos en aquellos pa- 
cientes con un centro respiratorio inestable, apneas o hipoventilación. En otras condiciones, los modos asistidos, solos o asociados con modos controlados, pueden ser utilizados con seguridad.

Se utilizará el ventilador que esté disponible en el servicio, sea este de flujo continuo o convencional de terapia intensiva. Se debe conocer bien las características técnicas del mismo (por ejemplo, criterio de ciclado por flujo en PSV, algoritmo de compensación de fugas, etc), para optimizar la relación y adaptación entre paciente y ventilador. Por último, debe tomarse en cuenta que una de las principales causas de falla en la VNI es la pérdida de la sincronía pacienteventilador $^{37}$.
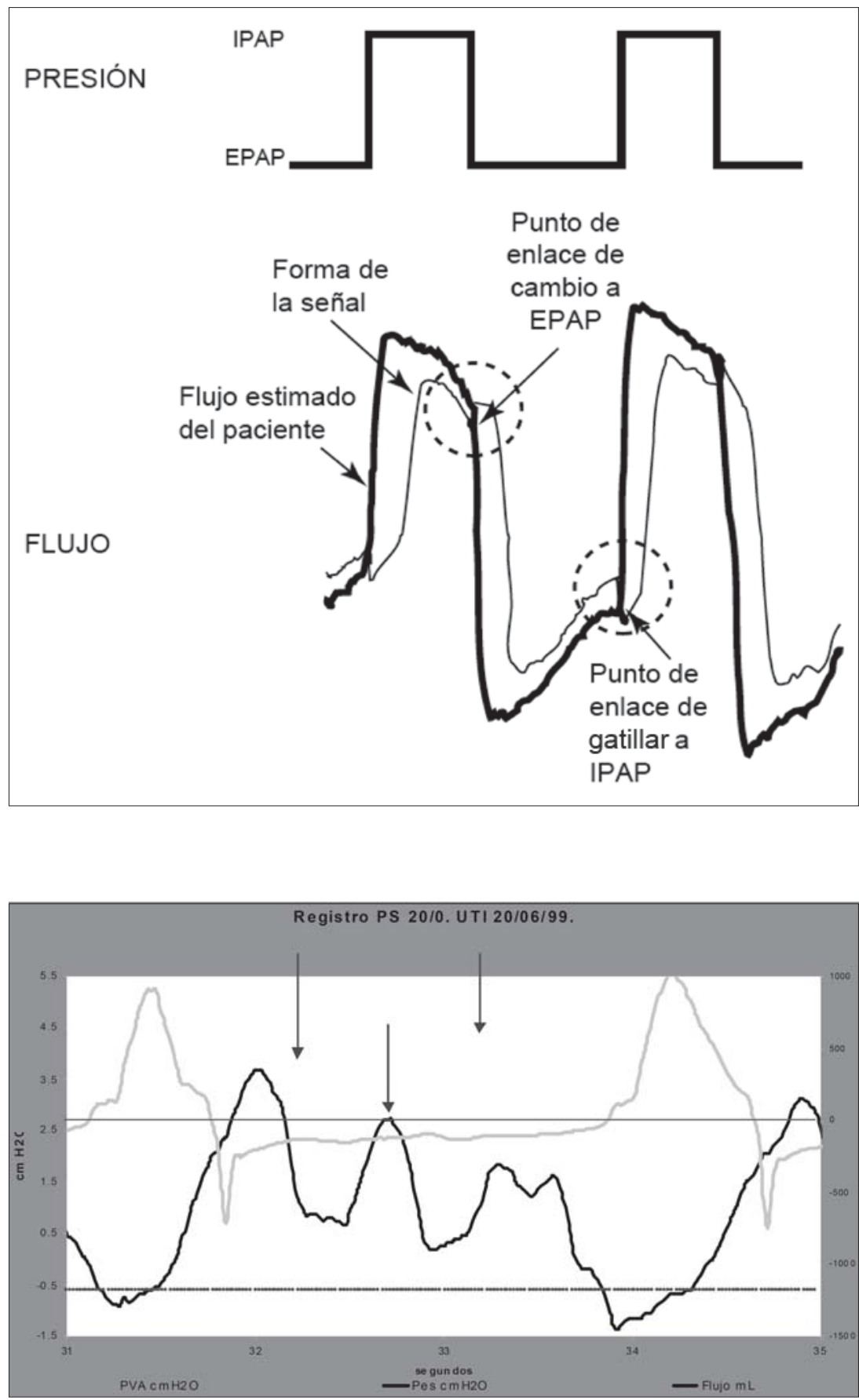

Figura 10. Auto Trak Shape Signal. Disparo inspiratorioespiratorio: este método realiza un seguimiento continuo de la onda de flujo inspiratoria y espiratoria del paciente. Se genera una señal digital que copia la onda de flujo original del paciente, con un retardo de 300 ms, y una caída del flujo inspiratorio de $15 \mathrm{~L} / \mathrm{min}$ en relación a la original del paciente. Cuando la frecuencia de flujo cruza la forma de señal digital, el ventilador cambia los niveles de presión pasando de IPAP a EPAP y viceversa. Dicho sistema permite un adecuado ajuste del ventilador ante los cambios bruscos del patrón respiratorio del paciente.
Figura 11. Medición de presión esofágica (negro) y flujo (gris) en un paciente con enfermedad pulmonar obstructiva crónica. El paciente está ventilado con presión de soporte (PSV), empleando una presión inspiratoria de $20 \mathrm{~cm} \mathrm{H}_{2} \mathrm{O}$, sin PEEP. Obsérvense los disparos ineficaces del paciente (flechas) en virtud de competir contra la PEEP intrínseca. En un período de 4 segundos, el paciente hizo 5 esfuerzos inspiratorios (Gentileza Dr. Guillermo Montiel). 


\section{Bibliografía}

1.- GRAY A, GOODACRE S, NEWBY D E, MASSON M, SAMPSON F, NICHOLL J. Noninvasive ventilation in acute cardiogenic pulmonary edema $\mathrm{N}$ Engl $\mathrm{J}$ Med 2008; 359: 142-51.

2.- PETER J, MORAN J, PHILLIPS HUGES J, GRAHAM $P$, BERSTEN A. Effect of non invasive positive pressure ventilation (NIPPV) on mortality in patients with acute cardiogenic oedema: a meta-analysis Lancet 2006; 367: 1155-63.

3.- CRANE S D, ELLIOT M W, GILLIGAN P, RICHARDS K, GRAY A J. Randomized controlled comparison of continuous positive airway pressure, bilevel non invasive ventilation, and standard treatment in emergency department patients with acute cardiogenic oedema. Emerg Med J 2004; 21: 155-61.

4.- NAVALESI P, FANFULlA F, FRIGERIO P, GREGORETTI C, NAVA S. Physiologic evaluation of non invasive mechanical ventilation with three types of masks in patients with chronic hypercapnic respiratory failure. Crit Care Med 200; 28: 1785-90.

5.- FERRIS B G, POLLARD D S. Effect of deep and quiet breathing on pulmonary compliance in man. J Clin Invest 1960; 39: 143-9.

6.- BENDIXEN H H, HEDLEY-WHYTE J, LAVER M B. Impaired oxygenation in surgical patients during general anaesthesia with controlled ventilation: a concept of atelectasis. N Eng J Med 1963; 269: 991-6.

7.- VISIK W D, FAIRLEY H B, HICKEY R F. The effects of tidal volume and end expiratory pressure on pulmonary gas exchange during anaesthesia. Anaesthesiology 1973; 39: 285-90.

8.- BACH J. Amyotrophic lateral sclerosis prolongation of life by noninvasive respiratory aids. Chest 2002; 122: $92-8$

9.- MARINI J J, CAPPS J S, CULVER B H. The inspiratory work of breathing during assisted mechanical ventilation. Chest 1985; 87: 612-8.

10.- HESS D R, KACMAREK R M. Essentials of mechanical ventilation. Chap 6, page 41-8, 1995.

11.- CALDERINI E, CONFALONIERI M, PUCCIO P G, FRANCAVILLA N, STELLA L, GREGORETTI C. Patient-ventilator asynchrony during noninvasive ventilation: the role of expiratory trigger. Intensive Care Med 1999; 25: 662-7.

12.- MACINTYRE N R. Respiratory function during pressure support ventilation. Chest 1986; 89: 677-83.

13.- APPENDINI L, PATESSIO A, ZANABONI S, CARONE M, GUKOV B, DONNER C F, et al. Physiologic effects of positive end-expiratory pressure and mask pressure support during exacerbations of chronic obstructive pulmonary disease. Am J Respir Crit Care Med 1994; 149: 1069-76.

14.- MACINTYRE N R, HO L I. Effects of initial flow rate and breath termination criteria on pressure support ventilation. Chest 1991; 99: 134-8.

15.- BROCHARD L, HARF A, LORINO H, LEMAIRE F. Inspiratory pressure support prevents diaphragmatic fatigue during weaning from mechanical ventilation. Am Rev Res Dis 1989; 139: 513-21.

16.- VAN DE GRAAFF W B, GORDEY K, DORNSEIF S E, DRIES D J, KLEINMAN B S, KUMAR P, et al. Pressure support: changes in ventilatory pattern and components of the work of breathing. Chest 1991; 100: 1082-9.

17.- BROCHARD L, MANCEBO J, WYSOKY M, LOFASO
F, CONTI G, RAUSS A, et al. Noninvasive ventilation for acute exacerbations of chronic pulmonary disease. N Engl J Med 1995; 333: 817-22.

18.- BROCHARD L, MOLLO J L, MANCEBO J, AMARO P, LEMAIRE F, HARF A. Comparison of the efficacy of inspiratory pressure support delivered by three ventilators. Am Rev Res Dis 1989; 139 (suppl): A 361.

19.- BROCHARD L, PLUSKWA F, LEMAIRE F. Improved efficacy of spontaneous breathing with inspiratory presssure support. Am Rev Res Dis 1987; 136: 4115.

20.- DÍAZ O, IGLESIA R, FERRER M, ZAVALA E, SANTOS C, WAGNER P, et al. Effects of noninvasive ventilation on pulmonary gas exchange and hemodynamics during acute hypercapnic exacerbations of chronic obstructive disease. Am J Respir Crit Care Med 1997; 156: 1840-5.

21.- FABRY B, GUTTMANN J, EBERHARD L, BAUER T, HABERTHÜR J, WOLFF G. An analysis of desychronization between the spontaneously breathing and ventilator during inspiratory pressure support. Chest 1995; 107: 1387-94.

22.- FERRERO G, ALTOBELLI L, LEBUS J. Ventilación no invasiva a presión positiva en la insuficiencia respiratoria aguda. PROATI, Módulo 3, Editorial Panamericana. 2001: 149-79.

23.- CHANG A A, STEINFELD A, GROPPER C, et al. Demand flow airway pressure release ventilation as partial ventilatory support mode: comparison with SIMV + PSV. Crit Care Med 1994; 22: 1431-7.

24.- SYDOW M, BURCAHRDI H, EPHRAIN E, ZIELMANN S, CROZIER T A. Long term effect of two different ventilatory modes in oxygenation in acute lung injury. Am J Respir Crit Care Med 1994; 149: $1550-6$.

25.- RASANEN J, CANE R D, DOWNS J B, HURST J M, JOUSELA I T, KIRBY R R, et al. Airway pressure release ventilation during acute lung injury. Crit Care Med 1991; 19: 1234-41.

26.- FERRERO G. Auto test: Pequeños grandes detalles en ventilación no invasiva. Revista Iberoamericana de Ventilación Mecánica No Invasiva 2004; 3: 40-3.

27.- VITACCA M, CLINI E, PAGANI M, BIANCHI A, AMBRONSINO N, ROSSI A. Physiologic effects of early administered mask proportional assist ventilation in patients with chronic obstructive pulmonary disease and acute respiratory failure. Crit Care Med 2000; 28: 1791-7.

28.- WYSOKI M, RICHARD J C, MESHAKA P. Noninvasive proportional assist ventilation compared with noninvasive pressure support ventilation in hypercapnic acute respiratory failure. Crit Care Med 2002; 30: 323-9.

29.- GAY P, HESS D, HILL N. Noninvasive proportional assist ventilation for acute respiratory insufficiency: comparison with pressure support ventilation. Am J Respir Crit Care Med 2001; 164: 1606-11.

30.- GEORGOPOULOS D, PRINIANAKIS G, KONDILI E. Bedside waveforms interpretation as tool to identify patient-ventilator asynchronies. Intensive Care Med 2006; 32: 34-47.

31.- HIRSCH C, KACMAREK R M, STANEK K. Work of breathing during CPAP and PSV imposed by the new generation ventilators: a lung model study. Respir Care 1991; 36: 815-28.

32.- GUREVITCH M J, GELMONT D. Importance of trigger sensitivity to ventilator response delay in 
advance chronic pulmonary obstructive disease with respiratory failure. Crit Care Med 1989; 17: 354-9.

33.- PRINIANAKIS G, KONDILI E, GEORGOPOULOS D. Effects of the flow waveform method of triggering and cycling on patient ventilator interaction during pressure support. Intensive Care Med 2003; 29: 19509.

34.- BATTISTI A, TASSAUZ D, JANSSENS J P, MICHOTTE J B, JABER S, JOILLIET P. Performance characteristics of 10 home mechanical ventilators in pressure-support mode. Chest 2005; 127: 1784-92.

35.- STELL I, GRAHAM P, LEE K, PONTE JOSE,
MOXHAM J. Noninvasive ventilator triggering in chronic obstructive pulmonary disease. Am J Respir Crit Care Med 2001; 164: 2092-7.

36.- ACOSTA B, DI BENEDETTO R, RAHIMI A, ACOSTA M F, CUADRA O, VAN NGUYEN A, et al. Hemodynamic effects of noninvasive bilevel positive airway pressure on patients with chronic congestive heart failure with systolic dysfunction. Chest 2000; 118: 1004-9.

37.- KACMARECK R M. NIPPV: patient-ventilator synchrony, the difference between success and failure? (Editorial). Intensive Care Med 1999; 25: 645-7. 